\title{
Selection of eyes or patients? Statistical trap
}

\section{¿Selección de ojos o pacientes? Trampa estadística}

\author{
Christian Fau ${ }^{1 *}$, Solange Nabzo ${ }^{1}$ and Veronica Nasabun ${ }^{2}$ \\ ${ }^{1}$ Ophthalmological Foundation 2020, Iberoamerican Cochrane Network; ${ }^{2}$ Universidad Andrés Bello, School of Nursing. Santiago, Chile
}

In clinical or epidemiological studies in ophthalmology, unlike other specialties, there is a problem in the selection of the sample, since the study subjects potentially have two interrelated sources of data, that is, two eyes that contribute to the study. Potentially one eye could be enrolled, or we could double our sample by enrolling both eyes. If a patient has information available for both eyes, why not using it?

The problem is that, probably, the findings in the left eye of an individual are generally more similar to those of the right eye of the same individual than those of the eye of another individual. This is due to multiple factors and is called inter-eye correlation. Common statistical techniques assume that the values of the observations are independent in each eye measured. In this case, when incorporating both eyes, this is not true. Currently, many articles do not consider this issue, and ignoring this fact can lead to a rejection of the study for publication.

In the literature there are mainly 4 different types of studies in ophthalmology, according to the selection criteria of the sample $\left.{ }^{1,2}: 1\right)$ subject design, in which the object of the analysis is the individual, for example, studies of strabismus or visual impairment; 2) pairedeye design, in which both eyes are incorporated and compared against each other; 3) one-eye design, in which only one eye of each patient is selected to be included in the study, and 4) two-eye design, in which some individuals contribute with one eye and others with two, or all with two eyes; in the latter, the two eyes can be, for example, randomized to the same treatment group, to different groups or to mixed groups.

According to the study by Lee, et al..$^{1}$ it is estimated that $13 \%$ of published clinical trials correspond to a subject design or individual analysis, which do not pose major design problems. Another $13 \%$ corresponds to a paired-eye design, which requires statistical analysis for paired data, so it does not present methodological problems either. Nevertheless, $48 \%$ corresponds to a one-eye design. This is the most used design, possibly because it is the simplest method, uses classical statistics and there is no inter-eye interference. The main problem with this method is that there is a potential waste of information that leads to less accurate estimates, since the other eye is available, often measured and then the information is not used. In some studies, in an attempt not to lose information from the second eye, an average calculation of the two eyes of those patients who have the two available records is used ${ }^{2}$. In addition, in these studies, special attention should be paid to clearly stating which method was used to select the eye included in the analysis. Lee ${ }^{1}$ estimated that, in $52 \%$ of published articles, the method is not clearly stated. The method can also be biased if

Correspondence:

${ }^{*}$ Christian Fau

Avd. Presidente Riesgo, 5157, Dep. 212 Las Condes

Date of reception: 20-06-2019

Date of acceptance: 05-09-2019

Santiago, Metropolitana, Chile

E-mail: chfauf@gmail.com

2604-1731/@ 2020 Sociedad Mexicana de Oftalmología. Published by Permanyer. This is an open creativecommons.org/licenses/by-nc-nd/4.0/).

DOI: 10.24875/RMOE.M20000096

Available online: 02-01-2020 Rev Mex Oftalmol (Eng). 2020;94(1):48-49 www.rmo.com.mx
C-ND license (http:// 
randomization was not used, for example, use of the worst or best eye, of the operated eye, the first eye with disease, only right or left eyes, or in some patients it was randomized and in others the eye that had the complete information was selected. Finally, the two-eye design is the most complex of all designs. Lee estimated that $19 \%$ of the published studies correspond to this design, and in none of the studies analyzed he found that the authors adjusted the sample size, and only in $39 \%$ of the studies the authors adjusted the statistical analysis through between-eye correlation. In the study by Murdoch ${ }^{2}$ this value was lower, of $11 \%$.

Analyses that do not consider eye correlation assume that we have more information than we really have. This leads to a falsely high degree of precision, an underestimation of the standard error, the confidence intervals become more precise and the differences between the two groups generate $p$-values falsely smalle $r^{2,3}$. If the authors do not have the possibility of performing more complex statistical analyses both for sample size calculation and for adjusting results correlation, then they should avoid this type of design, since they are at risk of rejection from a journal when sending an article for evaluation. Or, if the paper is accepted, it will be quickly discarded by readers due to internal validity errors and it will not generate any impact as a publication.

\section{Conflicts of interest}

The authors declare no conflicts of interest.

\section{Ethical disclosures}

Protection of human and animal subjects. The authors declare that the procedures followed were in accordance with the regulations of the relevant clinical research ethics committee and with those of the Code of Ethics of the World Medical Association (Declaration of Helsinki).

Confidentiality of data. The authors declare that they have followed the protocols of their work center on the publication of patient data.

Right to privacy and informed consent. The authors have obtained the written informed consent of the patients or subjects mentioned in the article. The corresponding author is in possession of this document.

\section{References}

1. Lee CF, Cheng ACO, Fong DYT. Eyes or Subjects: Are Ophthalmic Randomized Controlled Trials Properly Designed and Analyzed? Ophthalmology. 2012;119(4):869-72.

2. Murdoch IE, Morris SS, Cousens SN. People and eyes: statistical approaches in ophthalmology. Br J Ophthalmol. 1998;82(8):971-3.

3. Newcombe RG, Duff GR. Eyes or patients? Traps for the unwary in the statistical analysis of ophthalmological studies. $\mathrm{Br} \mathrm{J}$ Ophthalmol. 1987;71(9):645-6. 